\title{
Cold Dark Matter Cosmology Conflicts with Fluid Mechanics and Observations
}

\author{
Carl H. Gibson \\ University of California at San Diego, La Jolla, California, 92122-0411, USA \\ Email: cgibson@ucsd.edu
}

(Received September 17, 2006; accepted June 12, 2007

\begin{abstract}
Cold dark matter hierarchical clustering (CDMHC) cosmology based on the Jeans (1902) criterion for gravitational instability gives predictions about the early universe contrary to fluid mechanics and observations. Jeans neglected viscosity, diffusivity, and turbulence: factors that determine gravitational structure formation and contradict small structures (CDM halos) forming from non-baryonic dark matter particle candidates. From hydro-gravitational-dynamics (HGD) cosmology, viscous-gravitational fragmentation produced supercluster $\left(10^{46} \mathrm{~kg}\right)$, cluster, and galaxy $\left(10^{42} \mathrm{~kg}\right)$ mass clouds in the primordial plasma with the large fossil density $\left(\rho_{0}=3 \times 10^{-17} \mathrm{~kg} \mathrm{~m}^{-3}\right)$ of the first fragmentation at $10^{12} \mathrm{~s}$, and a linear and spiral clump morphology reflecting stretching-compression rates near vortex lines of the plasma turbulence at the $10^{13} \mathrm{~s}$ plasma-gas transition. Gas proto-galaxies fragmented into proto-globular-star-cluster mass $\left(10^{36} \mathrm{~kg}\right)$ clumps of proto-planet clouds, presently frozen as earth-mass $\left(10^{24-25} \mathrm{~kg}\right)$ Jovian planets of the baryonic dark matter, about 30,000,000 rogue planets per star. All stars form from these planets. They appear in planetary nebulae and are misinterpreted as dark energy. Observations contradict the CDMHCC prediction of large explosive Population III first stars at $10^{16} \mathrm{~s}$, but support the gentle formation of small Population II first stars at $10^{13} \mathrm{~s}$ in globular star-clusters from HGD.
\end{abstract}

Keywords: Cold Dark Matter, Gravitational Structure Formation, Dark Energy, Turbulence

\section{INTRODUCTION}

The Jeans (1902) acoustic criterion for self-gravitational structure formation is derived by linear perturbation stability analysis of the Euler fluid momentum equations with gravity, giving linear acoustic equations by neglecting diffusion, viscous forces and turbulence. Density $\rho$ is set to zero (the Jeans swindle) to derive a critical (Jeans) length scale $L_{J}=V_{S} /(\rho G)^{1 / 2}$, where $V_{S}$ is sound speed and $\mathrm{G}$ is Newton's gravitational constant, interpreted by Jeans and astrophysicists (e.g. Springel et al. 2005) as the only gravitational stability criterion, thus forbidding gravitational structures at scales smaller than $L_{J}$. Sir James Jeans (1929) claimed observational support for his theory is provided by spiral galaxies. He assumed hot gas from a (mysterious) central spinning source collapses to stars in such galaxies only when chilled by the coldness of space, thus reducing $L_{J}$.

This evidence weakened as better telescopes showed the "stars" observed were really globular star clusters (GCs) containing millions of stars. Observational and theoretical support for a central gas source in spiral galaxies failed to materialize. Modern fluid mechanics requires additional gravitational structure formation criteria. When viscosity, diffusion and turbulence are included in the analysis, a hydro-gravitational-dynamics (HGD) cosmology emerges that is in better agreement with recent observations of the early universe than CDM scenarios that assume collisionless fluids (Gibson 1996, 2000, 2004, 2005, 2006).

From HGD cosmology (Fig. 1), proto-galaxies and protogalaxy-clusters were formed by gravitational fragmentation of plasma at the Schwarz viscous scale $L_{S V}=(\gamma \nu / \rho G)^{1 / 2}$, where $\gamma$ is the rate-of-strain, $v$ is the kinematic viscosity, $\rho$ is the density, and $G$ is Newton's constant, preserving various parameters of these irreversible transitions as fluid mechanical fossils. Because the Kolmogorov scale $L_{K}=(v / \gamma)^{1 / 2}$, and because $\gamma /(\rho G)^{1 / 2} \approx 1$ for a flat universe, it follows that 
$L_{S V} \approx L_{N} \approx L_{K}$ for the conditions of proto-galaxy formation shown in Fig. 1. The first condensation was to form primordial fog particle (PFP) planets in Jeans mass proto-globular-star-cluster (PGC) clumps as the plasma proto-galaxies became gas with much smaller viscosity. Stars form as binaries when these planets merge in a binary cascade to form larger and larger mass Jovian planets (JPPs), now frozen as the baryonic (proton-neutron) dark matter. JPPs randomly dim supernovae in planetary nebulae, creating the now commonly accepted "dark energy" misconception (Gibson \& Schild 2007).

\section{DILEMMAS OF JEANS' THEORY}

The Jeans criterion presents major dilemmas for hot big bang cosmology in an expanding universe. In the $\mathrm{H}-\mathrm{He}$ plasma epoch, the Jeans scale of the plasma $L_{J}$ always exceeds the "horizon" scale of causal connection $L_{H}=c t$ because the enormous sound speed $V_{S}=c / 3^{1 / 2}$ is of order the speed of light $c$, where $t$ is the time, so plasma structures are forbidden. The primordial gas at transition has a Jeans mass of a million stars $\left(M_{J}=10^{36} \mathrm{~kg}\right), t=10^{13} \mathrm{~s}(300,000$ years), and $\mathrm{T}_{0}=4000 \mathrm{~K}$. Fragmentation in the primordial plasma occurs when the Schwarz viscous scale $L_{S V}$ matches the horizon scale $L_{H}$ at $10^{12} \mathrm{~s}$ (30,000 years), and in the primordial gas at the Jeans mass from heat transfer considerations and simultaneously at $L_{S V}$ to form Earth-mass primordial planets.

The Jeans mass $M_{J}=M_{J_{0}}\left(T / T_{0}\right)^{3 / 2} \rho / \rho_{0}$ from the Jeans criterion, so from Jeans' theory the first stars are large and explosive, Madau (2006), and can form only after a "dark ages" period of about $300 \mathrm{Myr}\left(10^{16} \mathrm{~s}\right)$ to permit sufficient cooling and density decrease. These Population III superstars rapidly disintegrate as dusty class II supernovas to re-ionize the gas and explain its absence in absorption spectra from early quasars, Mori and Umemura (2006). However, recent $\gamma$-ray spectra from blazars refute the intense high-energy starlight expected, Aharonian et al. (2006). The intergalactic medium is free of any high density of Pop-III photons that would scatter blazar $\gamma$-rays by pair production. Chemical searches also fail to detect the large amounts of stardust Pop-III superstars would have produced had they existed.

\section{HGD COSMOLOGY}

After the big bang and a fragmentational cascade to form protogalaxies (Fig. 1, Gibson 2006), HGD cosmology predicts primordial gas planets were the first collapsing gravitational objects. Superstars of CDM cosmology are not necessary to explain the missing gas since it was sequestered as planets. Some of the planets gently formed larger planets and small stars in dark proto-globular-clusters (PGCs) of dense, dark, cooling proto-galaxies (PGs). Freezing weakly collisional PGC clumps expanded by diffusion from PGs to form $>10^{21} \mathrm{~m}$ galaxy halos of baryonic dark matter (BDM), with diffusivity $D \sim 10^{27} \mathrm{~m}^{2}$ $\mathrm{s}^{-1}$ calculated from $L_{S D}$ and the size and $\rho$ of the halos.

Flat rotation curves observed outside the $10^{20} \mathrm{~m}$ spiral galaxy cores reflect this huge diffusivity. Luminous $>10^{20}$ $\mathrm{m}$ accretion disks and spiral patterns of star formation in globular-star-clusters (GCs) reflect torques developed in the chain-like morphology of the proto-galaxy-clusters as they split into spiral galaxies from the expansion of space. The tiny first stars still exist in GCs and dim proto-GCs that are bound by gravity and friction from the evaporated gas provided by heating of their trillion frozen gas planets.

\section{CDM COSMOLOGY}

Orthodox collisionless cosmology explains galaxy and galaxy cluster formation using "cold dark matter" (CDM): a postulated non-baryonic fluid of collisionless massive particles produced during the nucleosynthesis epoch with particle velocity $\mathrm{v}_{C D M}<c$. Based on Jeans theory and collisionless N-body simulations, it is assumed deus ex machina, Binney \& Tremaine (1987), that gravitationally bound CDM chunks form and merge gravitationally like point mass $\mathrm{N}$-bodies are forced to do in computer simulations. Large mergers ("halos") of CDM chunks then serve as growing baryon bottles that guide the gravitational formation of larger structures by hierarchical clustering (CDMHC) for $300 \mathrm{Myr}$ dark ages without stars and billions of years without galaxies or galaxy clusters.

Pop-III superstars form as the gas cools by radiation and promptly explode in the merging CDM-halos, Mori et al. (2004). Jupiter mass gas planets are ruled out by the Jeans criterion because they require an impossible cooling of their $\mathrm{H}-\mathrm{He}$ gas to $0.004 \mathrm{~K}$ to permit Jeans instability. However, Jovian planets circling the sun in outer orbits and now detected in great multitudes in tight orbits around other stars, Lovis et al. (2006), contradict the Jeans acoustic criterion and support the basic tenet of HGD that all the hot gas after transition fragments into dense PGC globular star cluster mass clouds of Earth mass PFP primordial fog particles.

How can the postulated CDM chunks remain gravitationally bound and merge to form larger chunks? Because fundamental particles of CDM are assumed to be collisionless and the chunks are not point masses but must occupy finite volumes, CDM chunks have no physical mechanisms to stick to each other on collision or resist tidal forces $F_{T I D A L}=d_{C D M} G m_{C D M} M_{C D M} / r^{3}$, where $d_{C D M}$ is the diameter of CDM chunks of mass $m_{C D M}$ attracted by a larger chunk of mass $M_{C D M}$ at distance r.

The tidal distortion $x_{\text {TIDAL }}$ per free fall oscillation is $x_{T I D A L} / d_{C D M}=(t / \tau)^{2}\left(D_{C D M} / r\right)^{3} M_{C D M} / 2 m_{C D M}>1$ 
- Small $m_{C D M} \ll M_{C D M}$ chunks are torn to smaller and smaller masses $m_{C D M} \rightarrow m_{C D M}$-particle by any larger masses $M_{C D M}$ in a few free fall times $\tau=(\rho G)^{-1 / 2}$ (Fig. 2), contradicting the hierarchical merging process postulated in CDM cosmology. Chunks of CDM of any size will not remain gravitationally bound but will expand indefinitely by diffusion. The CDMHC hypothesis is therefore false. Observations show no galaxies with cusplike central concentrations of density reflecting the collisionless core collapse expected for CDM halos of CDM chunks.

Gravitationally bound CDM chunks are claimed (Binney \& Tremaine 1987, chapter 4) to have large stability

periods $\tau_{C D M}=\tau N / \ln N$ much longer than $\tau$ if $\mathrm{N}$ is large, where $\mathrm{N}$ is the number of CDM particles. The claim is unwarranted. Any collection of collisionless particles with any velocities will grow in size indefinitely by diffusion. Assume a spherical CDM-chunk initially with perfectly cold, motionless, particles. All particles fall toward the center of the sphere arriving at time $t \approx \tau$ as the density of the shrinking sphere rapidly increases, the collisionless assumption fails, the particles exchange momentum and energy, and diffusion increases the size of the chunk to a scale $L_{S D}=\left(D^{2} / \rho G\right)^{1 / 4}$ larger than its initial size (Fig. 3). The free fall time $\tau$ increases as the CDM-chunk size increases.

Galaxies of stars in apparent virial equilibrium are claimed, Binney \& Tremaine (1987), as prime examples of relatively permanent gravitationally-bound $\left(\tau_{\text {galaxy }} \sim \tau N / \ln N\right)$ collisionless systems. However, stars are actually not collisionless because on average 30 million planets surround each star as the interstellar medium from which stars are formed. The planets supply gas and friction in response to any rapid relative motions, and reveal themselves as contrails of stars (as in the Tadpole, Mice, and Antenna systems) formed in galaxy baryonic dark matter halos when galaxies frictionally merge, Gibson \& Schild $(2003,2007)$, Gibson (2006).

Schild (1996) has shown by observations of quasar microlensing twinkling frequencies that the lensing galaxy mass is dominated not by stars but by planets, which he proposed as the galaxy missing mass. Numerous claims that the collisionless tidal tails of computer simulations of galaxy mergers have been observed in nature are therefore false. Star trails between merging and separating galaxies are never collisionless tidal tails but evidence of galaxies triggering star formation as they move through each other's highly combustible BDM halos.

Diffusivity $D=L \mathrm{v}$ in a gas is mean free path $L_{M F P}=m_{\text {particle }} / \rho_{\text {gas }} \sigma_{\text {collision }}$ times the particle velocity $\mathrm{v}_{\text {particle }}$, so whatever fundamental particle emerges as that of the unknown (neutrino or neutrino-like) non-baryonic dark matter (NBDM) will certainly have a very large $L_{M F P}$ and large diffusivity $D_{N B D M}$ since the collision cross sectional area $\sigma_{N B D M}$ is small $\left(<10^{-40} \mathrm{~m}^{2}\right)$ and the particle velocity $\mathrm{v}_{N B D M} \sim c$ large.

From the size and density of galaxy clusters and the Schwarz diffusive scale $L_{S D}=\left(D^{2} / \rho G\right)^{1 / 4}$, the NBDM particle mass appears to be about $10^{-35} \mathrm{~kg}$, Gibson (2000), with diffusivity $D_{N B D M} \approx 10^{30} \mathrm{~m}^{2} \mathrm{~s}^{-1}$ and a total NBDM mass about 30 times that of all baryons. With these values, the NBDM diffuses to $L_{S D}$ scales somewhat larger than the horizon scale $c t$ during the plasma epoch and begins fragmentation at galaxy cluster halo scales of about $10^{23} \mathrm{~m}$ after the transition of plasma to gas. In the hot plasma period between $10^{2} \mathrm{~s}$ and $10^{11} \mathrm{~s}$ when energy dominated mass NBDM particles coupled to the plasma to dominate momentum transfer in both fluids, preventing both turbulence and gravitational structure formation. A mechanism like the high temperature MSW scattering of neutrinos by electrons is anticipated, Mikheyev \& Smirnov (1985), Wolfenstein (1978).

No cosmological dilemmas arise if collisional, nonlinear fluid mechanics is applied to cosmology rather than the unrealistic simplifications of Jeans (1902) and CDMHC. Self-gravitating fluids are absolutely unstable at density minima and maxima and form structures unless prevented by fluid forces or diffusion. Fossil turbulence ${ }^{*}$ from inflation preserves patterns of the big bang turbulence, Gibson (2004).

Only viscous and turbulence forces were relevant in the primordial plasma until buoyancy forces of the first selfgravitational structures (at $10^{12} \mathrm{~s}$ ) appeared to inhibit large scale turbulence ${ }^{\dagger}$ and to preserve the plasma density ( $\rho_{0}$ $\left.\sim 3 \times 10^{-17} \mathrm{~kg} \mathrm{~m}^{-3}\right)$ and rate-of strain $\left(\gamma_{0} \sim 10^{-12} \mathrm{rad} \mathrm{s}^{-1}\right)$ as fossils of the turbulence. Diffusion dominates gravity for the NBDM in the plasma epoch since $L_{S D}>L_{H}=c t$ during this period (Gibson 2000).

Coupling to the plasma ceased soon after $10^{11} \mathrm{~s}$. Condensation on density maxima was inhibited by the rapid expansion rate of the early universe, so the first gravitational structures were formed by fragmentation at

\footnotetext{
* Fossil turbulence is defined as a fluctuation in any hydrophysical field produced by turbulence that persists after the fluid ceases to be turbulent at the scale of the perturbation.

Turbulence is defined as an eddy-like state of fluid motion where the inertial vortex forces of the eddies $\overrightarrow{\mathrm{v}} \times \vec{\omega}$ are larger than any other forces that tend to damp the eddies, $\overrightarrow{\mathrm{V}}$ is the velocity and $\vec{\omega}$ is the vorticity. Turbulence by this definition always cascades from small scales to large.
} 
density minima when the viscous Schwarz scale $L_{S V}=(\gamma v / \rho G)^{1 / 2}>L_{H}$ decreased to less than $L_{H}=c t$ at $10^{12} \mathrm{~s}$, forming voids and clouds of plasma when viscous forces matched those of gravity, where $\gamma_{0} \sim 1 / t_{0}$ is the rate of strain $\left(10^{-12} \mathrm{~s}^{-1}\right)$ and $v$ is the kinematic viscosity $\left(4 \times 10^{26} \mathrm{~m}^{2} \mathrm{~s}^{-1}\right)$

Substituting these values gives $L_{S V} \approx L_{H}=3 \times 10^{20} \mathrm{~m}$ at $\mathrm{t}_{0}=10^{12} \mathrm{~s}$ with plasma mass $\sim 10^{45} \mathrm{~kg}$ corresponding to that of a galaxy supercluster. The horizon scale Reynolds number $\operatorname{Re}_{H}=c^{2} t / v \sim 250$ for the plasma was only slightly above critical, so the turbulent and viscous Schwarz scales $L_{S T}=\varepsilon^{1 / 2} /(\rho G)^{3 / 4} \approx L_{S V}$ were nearly identical. The viscous dissipation rate $\varepsilon$ was $\sim 800 \mathrm{~m}^{2} \mathrm{~s}^{-3}$. Patterns of big bang fossil turbulence that triggered the fragmentation are preserved by the cosmic microwave background, Gibson (2005).

\section{OBSERVATIONS}

Gravitational fragmentation of the plasma continued to smaller scales, forming protogalaxy mass clouds $\left(10^{42} \mathrm{~kg}\right)$ prior to gas formation, with density $\rho_{0}$ and an initial protogalaxy size $3 \times 10^{19} \mathrm{~m}(1 \mathrm{kpc})$. Density minima and maximum rates of strain $\gamma$ provide sites for fragmentation near vortex lines of the weak turbulence, giving dim straight chains of $6 \times 10^{19} \mathrm{~m}$ protogalaxy clumps (Fig. 4) and spiral clusters of protogalaxy clumps as observed by the Hubble Ultra Deep Field, Elmegreen et al. (2005).

Direct numerical simulations of turbulence, Nomura and Post (1998), show that the maximum $\gamma$ values required for gravitational structure formation by fragmentation occur near turbulence vortex lines and at the end of vortex tubes (Fig. 5). The pressure Hessian $\Pi_{i j}=\partial^{2} p / \partial x_{i} \partial x_{j}$ describes the complex non-local interactions of the vorticity $\vec{\omega}$ and the rate of strain tensor $S_{i j}=\left(\partial \mathrm{v}_{\mathrm{i}} / \partial \mathrm{x}_{\mathrm{j}}+\partial \mathrm{v}_{\mathrm{j}} / \partial \mathrm{x}_{\mathrm{i}}\right) / 2$, resulting in maximum values of $\mathbf{S}$ eigenvalues $\left(\gamma_{i j}\right)_{\text {eigen }}=(\alpha, \beta, \gamma) ; \alpha \geq \beta \geq \gamma$ along the vortex lines $\left(\gamma_{i j}\right)_{\text {eigen }}=(+,-,-)$ but with the average $\beta>0$ dominated by stretching in two directions near the ends $\left(\gamma_{i j}\right)_{\text {eigen }}=(+,+,-)$. All the eigenvalues are the same magnitude because $\alpha+\beta+\gamma=0$ from the continuity equation and incompressibility. Thus, two types of gravitational fragmentation regions are expected from turbulence induced stretching; the strain dominated vortex line regions giving linear chains of protogalaxy clumps, and the $\Pi$ and spin dominated ends of vortex tubes giving clump clusters of protogalaxies with a spiral pattern. The resulting protogalaxy morphology expected from HGD is observed, as shown in Fig. 5. The constant $10^{20} \mathrm{~m}$ viscous- gravitational scale of all protogalaxies at plasma-gas transition is termed the Nomura scale $L_{N}$.

The Schwarz viscous scale of the $4000 \mathrm{~K}$ hot gas at $10^{13} \mathrm{~s}$ was about $10^{14} \mathrm{~m}$ giving a small-planet mass of $10^{24-25} \mathrm{~kg}$. Simultaneous fragmentations at $10^{36} \mathrm{~kg}$ were from radiative heat transfer density changes at the Jeans scale $L_{J}$, not by the Jeans (1902) mechanism. The viscous dissipation rate $\varepsilon$ was a very gentle $\sim 10^{-12} \mathrm{~m}^{2} \mathrm{~s}^{-3}$.

Gravitational condensation of matter first began in the universe as planetary cloud collapse with $\tau_{0}=\left(\rho_{0} G\right)^{-1 / 2}$ in proto-globular-clusters (PGCs). The collapse time $\tau_{0}$ was $3 \times 10^{13} \mathrm{~s}(600,000$ years $)$ which is also the time to first star formation in the tidally agitated PGC cores near protogalaxy centers, not the 300 million dark ages years $\left(10^{16} \mathrm{~s}\right)$ predicted by CDMHC after assembly of CDM halos and cooling of the gas to permit the first stars as Population III superstars by the Jeans criterion. Recent star formation occurs in the strongly agitated PGCs of galaxy accretion disks, as shown in Fig. 6 and GALEX ultraviolet galaxy images, Gil de Paz et al. (2005). When strongly agitated, the PFP planets of PGCs form detectable massive clumps termed "globulettes" with total mass larger than the large (O and B) stars they produce, Gahm et al. (2007). Spiral structure in galaxies reflects PGC star trails of spiral shaped gravitational accretion, not Lindblad-Lin-Shu density waves.

\section{CONCLUSIONS}

The standard $\Lambda \mathrm{CDM}$ cosmology based on collisionless fluid mechanics, cold dark matter and dark energy is obsolete and should be abandoned because it gives predictions contrary to observations, and relies on many faulty assumptions. Hydro-gravitational-dynamics star formation and cosmology predictions give protosupercluster to protogalaxy formation in the plasma epoch $\left(10^{12}\right.$ to $\left.10^{13} \mathrm{~s}\right)$ by fragmentation triggered by density patterns of big bang turbulent mixing, preserved as the first fossils of turbulence, and positive straining along vortex lines of the weaker plasma turbulence (Fig. 1). Evidence of protogalaxy formation in clump chains (Fig. 4) and in spiral clump clusters (Fig. 5) is reported from HST/ACS images at Nomura (Kolmogorov) scales $10^{20} \mathrm{~m}$ with patterns reflecting those computed for turbulence at maximum and average stretching rates, Nomura and Post (1998). GALEX UV images (Fig. 6) reveal previously invisible PGC dark matter accretion disks.

From HGD, small stars formed in the protogalaxies from $10^{24-25} \mathrm{~kg}$ planets (PFPs) fragmented at Schwarz viscous scales $L_{S V}$ in Jeans mass $10^{36} \mathrm{~kg}$ clumps (PGCs) immediately after the $10^{13} \mathrm{~s}$ plasma to gas transition. The 
large baryonic density $\rho_{0}=3 \times 10^{-17} \mathrm{~kg} \mathrm{~m}^{-3}$ and large strain-rate $\gamma_{0} \approx 10^{-12} \mathrm{~s}^{-1}$ of the $10^{12} \mathrm{~s}$ protosupercluster fragmentation is fossilized by the density of globular star clusters and the masses of the frozen planets. Gentle motions prevailing at the $10^{13} \mathrm{~s}$ time of first stars made possible the small star sizes. The interstellar medium and the baryonic dark matter are metastable proto-globular-starclusters of frozen small planets, as predicted by the Gibson (1996) HGD cosmology and observed by the Schild (1996) quasar microlensing. These planets give a systematic dimming error when small stars die in planetary nebulae. This has been misinterpreted as dark energy with a cosmological constant $\Lambda \neq 0$, Gibson \& Schild (2007).

CDM hierarchical clustering cosmology gives gravitationally bound structures forming first at small scales and clustering by impossible fluid mechanics (Figs 2\&3). These structures gradually form stars that are much too large and too late, and that explode to form light that is found not to exist by the Aharonian et al. 2006 blazar gamma ray observations. Galaxies and galaxy clusters are observed at times earlier than predicted by CDMHC cosmology, but as expected from HGD cosmology.

\section{REFERENCES}

Aharonian, F. et al. (2006). A low level of extragalactic background light as revealed by $\gamma$-rays from blazers, Nature, 440, 1018-1021.

Binney, J. and Tremaine, S. (1987). Galactic Dynamics, Princeton University Press.

Elmegreen, D.M., Elmegreen, B.G., Rubin, D.S., Schaffer, M.A. (2005). Galaxy morphologies in the Hubble Ultra Deep Field: Dominance of linear structures at the detection limit, ApJ, 631:85-100.

Jeans, J. H. (1902). The stability of a spherical nebula, Phil. Trans., 199 A, 0-49

Jeans, J.H. Sir (1929). Astronomy and Cosmogeny, 2nd Ed., Cambridge University Press.

Gahm, G. F. et al. (2007). Globulettes as seeds of brown dwarfs and free-floating planetary-mass objects, $A J$, 133, 1795-1809.

Gibson, C.H. (1996). Turbulence in the ocean, atmosphere, galaxy and universe, Appl. Mech. Rev., 49, no. 5, 299315.

Gibson, C.H. (2000). Turbulent mixing, diffusion and gravity in the formation of cosmological structures: The fluid mechanics of dark matter, J. Fluids Eng., 122, 830-835.

Gibson, C.H. (2004). The first turbulence and the first fossil turbulence, Flow, Turbul. and Combust., 72, 161179.

Gibson, C.H. (2005). The first turbulent combustion, Combust. Sci. and Tech., 177: 1049-1071.
Gibson, C.H. (2006). The fluid mechanics of gravitational structure formation, http://xxx.lanl.gov/astroph/0610628.

Gibson, C. H. and Schild, R. E. (2003). Interpretation of the Tadpole VV29 merging galaxy system using hydro-gravitational theory. http://xxx.lanl.gov/ astroph/0210583.

Gibson, C.H. and Schild, R.E. (2003). Interpretation of the Helix planetary nebula using hydro-gravitational theory, http://xxx.lanl.gov/astro-ph/0306467.

Gibson, C.H. and Schild, R.E. (2007). Interpretation of the Helix planetary nebula using hydro-gravitationaldynamics: planets and dark energy, http://xxx.lanl.gov/ astro-ph/0701474.

Gil de Paz, A., Madore, B., et al. (2005). Discovery of an extended ultraviolet disk in the nearby galaxy NGC 4625, ApJL, 627, L29-L32.

Lovis, C. et al. (2006). An extrasolar planetary system with three Neptune-mass planets, Nature, 441, 3-5309.

Madau, P. (2006). Trouble at first light, Nature, 440, $100 z 1003$.

Mikheyev, S., Smirnov, A. (1985). Sov. J. Nucl. Phys., 42, 913.

Mori, M. \& Umemura, M. (2006). The evolution of galaxies from primeval irregulars to present-day ellipticals, Nature, 440, 644-647.

Mori, M., Umemura, M. \& Ferrara, A. (2004). The nature of Lya blobs: supernova dominated primordial galaxies, ApJ, 613, L97-L100.

Nomura, K.K. \& Post, G.K. (1998). The structure and dynamics of vorticity and rate of strain in incompressible homogeneous turbulence, J. Fluid Mech., 377, 65-97.

Schild, R.E. (1996). Microlensing variability of the gravitationally lensed quasar Q0957 561 A,B. ApJ, 464, 125-130.

Springel, V. et al. (2005). Simulations of the formation, evolution and clustering of galaxies and quasars, Nature, 435, 629-636.

Wolfenstein, L. (1978), Phys. Rev. D, 17, 2369. 


\section{FIGURES}

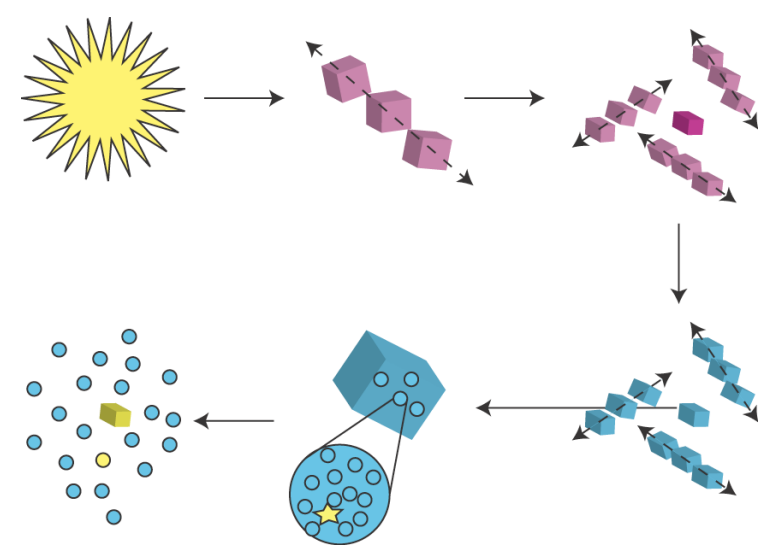

Fig. 1- Gravitational structure formation according to HGD cosmology (Gibson 2006). Proto-supercluster-voids form at minimum density points along vortex lines of weak turbulence in the hot plasma at $10^{12} \mathrm{~s}$ (top center). Fragmentation continues to $10^{13} \mathrm{~s}$ when plasma proto-galaxies turn to gas (right top bottom). These fragment into proto-globular-star-cluster (PGC) clouds of primordial-fog-particle planets (PFPs) that form the first stars (bottom center). The freezing PGCs diffuse out of the galaxy core to form its baryonic dark matter halo (bottom left).

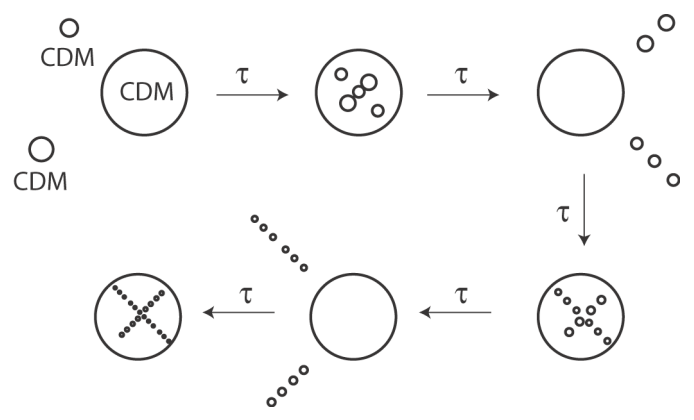

Fig. 2- Three hypothetical CDM chunks interact gravitationally. Because they have no sticking mechanism to hold them together, the collisionless CDM chunks are shredded to atoms in a few free fall times $\tau$ by tidal forces and cannot grow by CDM hierarchical clustering.

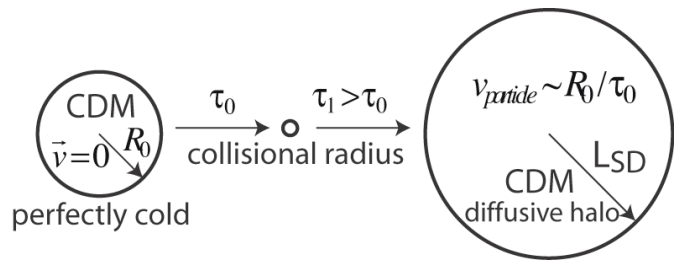

Fig. 3- A perfectly cold chunk of CDM with motionless particles collapses in a free fall time $\tau_{0}$ to a size small enough for the collisionless assumption to fail. The "chunk" then expands to size $L_{S D}$ with particle kinetic energies determined by the initial gravitational potential energy. 
Carl H. Gibson / JAFM, Vol. 1, No. 2, pp. 1-8, 2008.

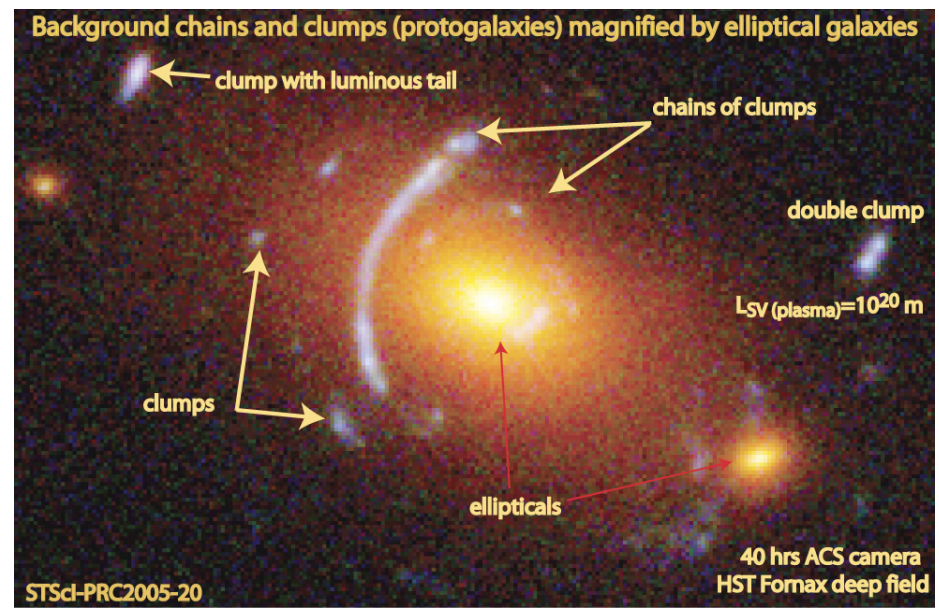

Fig. 4- Elliptical galaxies magnify light from the first galaxies, which are in linear structures reflecting viscousgravitational formation along vortex lines of the weakly turbulent plasma just before transition to gas (Fig. 1). Star formation is triggered in the BDM between the $2 \mathrm{kpc}\left(6 \times 10^{19} \mathrm{~m}\right)$ protogalaxies (clumps).

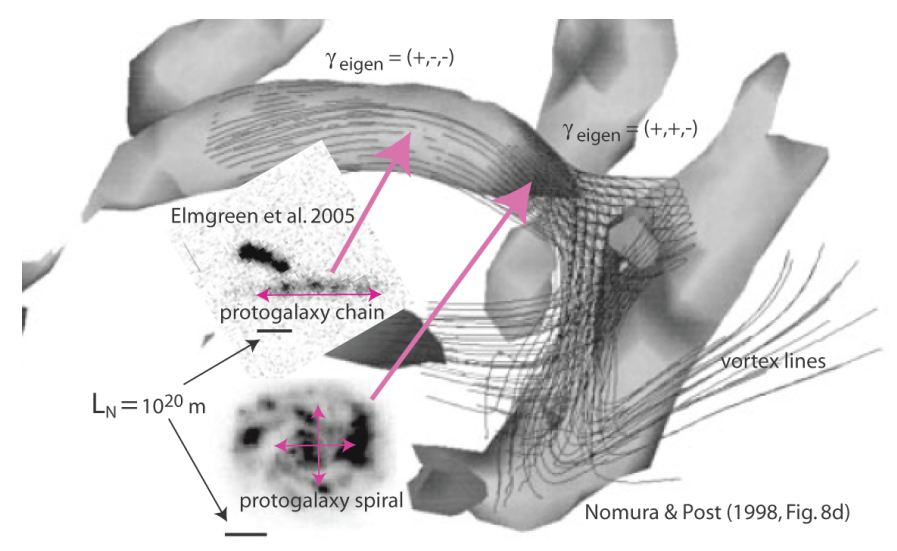

Fig. 5- Direct numerical simulations of turbulence by Nomura \& Post (1998) show maximum stretching rate $\alpha$ occurs on vortex lines where $\left(\gamma_{i j}\right)_{\text {eigen }}=(+,-,-)$, but the average stretching rate $\alpha \approx \beta>0$ comes from the end of vortex tubes where $\left(\gamma_{i j}\right)_{\text {eigen }}=(+,+,-)$. From HGD, protogalaxy formation in the turbulent plasma is triggered where $\alpha$ is large, which can explain the clump-chains and clump-spirals observed by Elmgreen et al. (2005). The Nomura scale $L_{N} \approx 10^{20} \mathrm{~m}$ is that of central bulge regions of most spiral galaxies, suggesting these are fossils of their protogalaxy clump origins at the plasma Kolmogorov scale $L_{K} \approx L_{N}$ just before transition to gas. 
Carl H. Gibson / JAFM, Vol. 1, No. 2, pp. 1-8, 2008.

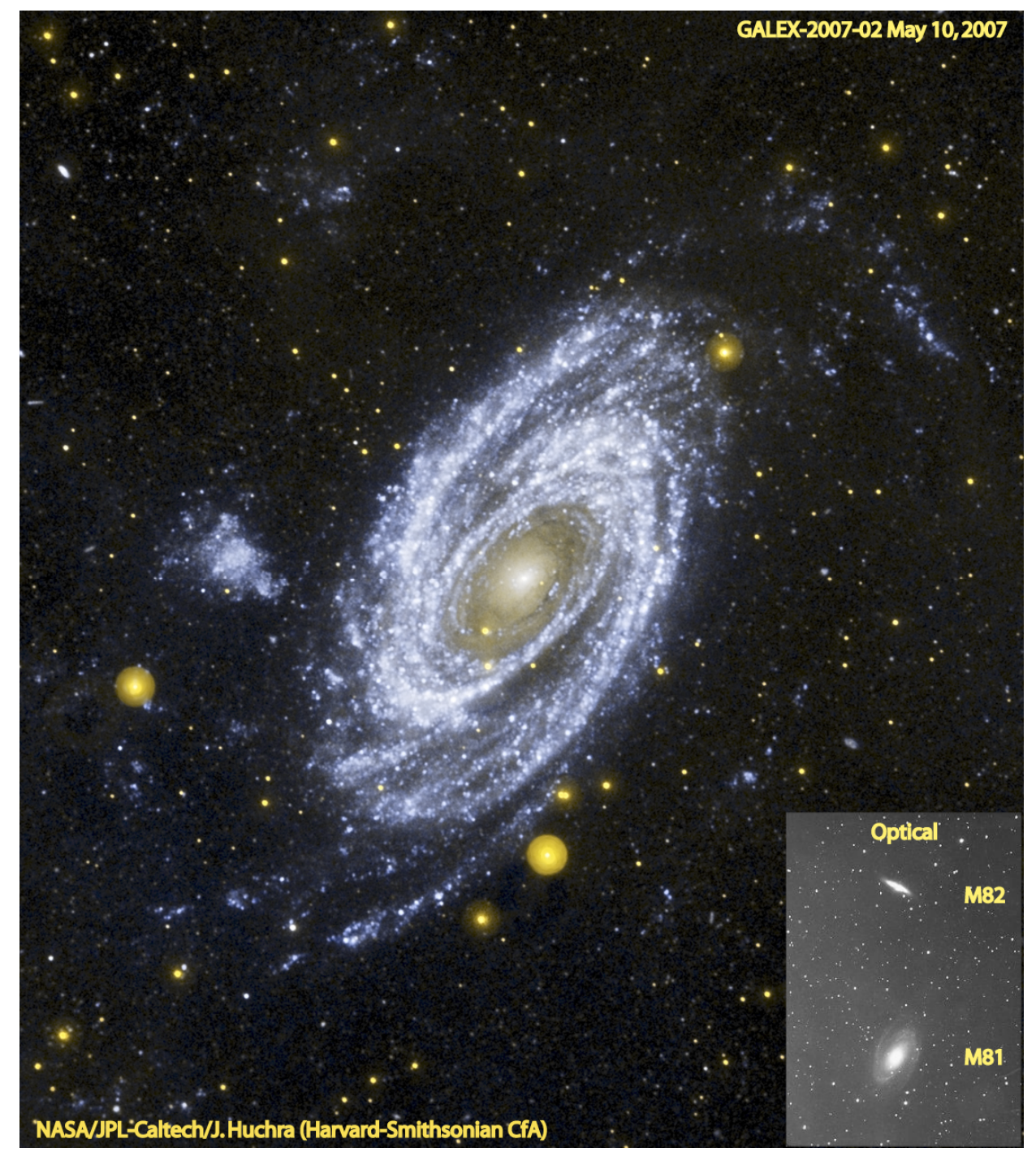

Fig. 6- Spiral galaxy M81 reveals its dark-matter accretion disk in the ultraviolet (GALEX satellite) that is invisible from optical telescopes on earth (lower right). Point-like blue disk objects are not stars but million-solarmass proto-globular-star-clusters (PGCs) illuminated by a few very hot young short-lived (O and B) stars and their ionized bubbles triggered into formation by viscous and tidal stresses of M81 separated from M82 by the expansion of the universe. Strong agitation of the trillion dark-matter PFP planets per PGC leads to large stars with short lives. Weak agitation produced the ancient small stars of the central core with the Kolmogorov (Nomura) scale $L_{K} \approx L_{N}$ of the plasma epoch. The baryonic dark-matter halo is comprised of PGCs diffused from proto-galaxy central cores of M81 and M82, and occupies a cylinder larger than the region shown by the optical insert (lower right). The non-baryonic dark matter has diffused about ten times larger to form the galaxy cluster halo $\geq 10^{22} \mathrm{~m}$. Dust trails on the face of the central core reflect spiral accretion in $\mathrm{O}$ and $\mathrm{B}$ supernovae stardust. 